\title{
Size-dependent interaction of silica nanoparticles with lysozyme and bovine serum albumin proteins
}

\author{
Indresh Yadav, ${ }^{1}$ Vinod K. Aswal, ${ }^{1}$ and Joachim Kohlbrecher ${ }^{2}$ \\ ${ }^{1}$ Solid State Physics Division, Bhabha Atomic Research Centre, Mumbai 400 085, India \\ ${ }^{2}$ Laboratory for Neutron Scattering, Paul Scherrer Institut, CH-5232 PSI Villigen, Switzerland
}

(Received 21 March 2016; published 2 May 2016)

\begin{abstract}
The interaction of three different sized (diameter 10, 18, and $28 \mathrm{~nm}$ ) anionic silica nanoparticles with two model proteins - cationic lysozyme [molecular weight (MW) $14.7 \mathrm{kDa}$ )] and anionic bovine serum albumin (BSA) (MW $66.4 \mathrm{kDa}$ ) has been studied by UV-vis spectroscopy, dynamic light scattering (DLS), and small-angle neutron scattering (SANS). The adsorption behavior of proteins on the nanoparticles, measured by UV-vis spectroscopy, is found to be very different for lysozyme and BSA. Lysozyme adsorbs strongly on the nanoparticles and shows exponential behavior as a function of lysozyme concentration irrespective of the nanoparticle size. The total amount of adsorbed lysozyme, as governed by the surface-to-volume ratio, increases on lowering the size of the nanoparticles for a fixed volume fraction of the nanoparticles. On the other hand, BSA does not show any adsorption for all the different sizes of the nanoparticles. Despite having different interactions, both proteins induce similar phase behavior where the nanoparticle-protein system transforms from one phase (clear) to two phase (turbid) as a function of protein concentration. The phase behavior is modified towards the lower concentrations for both proteins with increasing the nanoparticle size. DLS suggests that the phase behavior arises as a result of the nanoparticles' aggregation on the addition of proteins. The size-dependent modifications in the interaction potential, responsible for the phase behavior, have been determined by SANS data as modeled using the two-Yukawa potential accounting for the repulsive and attractive interactions in the systems. The protein-induced interaction between the nanoparticles is found to be short-range attraction for lysozyme and long-range attraction for BSA. The magnitude of attractive interaction irrespective of protein type is enhanced with increase in the size of the nanoparticles. The total (attractive+repulsive) potential leading to two-phase formation is found to be more attractive for larger sized nanoparticles. The nanoparticle aggregates are characterized by mass fractal.
\end{abstract}

DOI: 10.1103/PhysRevE.93.052601

\section{INTRODUCTION}

The study of the interaction of the nanoparticles with proteins has attracted considerable attention recently, evoked in part by the practical importance of their conjugates and in part to understand their complex interaction mechanism [1-4]. The potential applications of nanoparticleprotein conjugates include biosensing, drug delivery, imaging, and novel functional materials [3-6]. The important interactions that involve and govern the phase behavior of nanoparticle-protein systems are van der Waals attraction, steric repulsion, and electrostatic attraction or repulsion and depletion [7-9]. Although the well-established DerjaguinLandau-Verwey-Overbeek (DLVO) theory combining attractive van der Waals and repulsive electrostatic double-layer interactions has successfully been used for many colloidal systems, this theory cannot be applied when non-DLVO interactions (e.g., depletion, steric) become significant [8-11]. Further for nanoparticle-protein systems, the complexity of their interaction increases due to site-specific interaction of proteins with the nanoparticles arising from nonuniform charge distribution at the protein surface $[1,7]$. In the case of charged nanoparticles, the adsorption of proteins (core-shell structure) may occur irrespective of their charge nature [1214]. The oppositely charged proteins adsorb strongly on the nanoparticles and often result in protein-mediated aggregates of the nanoparticles $[15,16]$. The adsorption of similarly charged proteins has also been observed due to site-specific interaction of protein with the nanoparticles $[13,17,18]$. There are also nanoparticle-protein systems where the absence of site-specific adsorption (nonadsorption) induces depletion interaction and thereby can modify their stability and resultant structure [19-21]. The interaction of charged nanoparticles with model proteins has been studied under varying solution conditions. These studies show that type of protein (cationic vs anionic), solution conditions (e.g., ionic strength, $p \mathrm{H}$ ), and presence of additives (surfactant, polymer) play an important role in deciding the phase behavior of nanoparticle-protein systems [22-26].

We have earlier studied the differences in phase behavior of anionic silica nanoparticles with two model proteinscationic lysozyme and anionic BSA [22,25]. Both proteins can render the same kind of phase behavior where nanoparticleprotein systems transform from one phase (monodisperse) to two phase (nanoparticle aggregation) as a function of protein concentration. The differences in the interactions of the nanoparticles with two proteins are reflected in the much higher value of protein concentration required for BSA than lysozyme in inducing aggregation in the respective nanoparticle-protein system. The aggregation of the nanoparticles irrespective of the charge nature of protein indicates the nanoparticle-protein systems undergoing from repulsive to attractive behavior. The interactions between the nanoparticles have successively been modeled by combining electrostatic repulsion with short-range attraction for the lysozyme protein adsorption and long-range depletion attraction in the case of nonadsorption of BSA protein. The understanding of these interactions eventually decides the phase behavior and resultant structures [7]. The characteristics of the nanoparticles and proteins (size, shape, charge, etc.) as well as solution 
conditions ( $p \mathrm{H}$, ionic strength, temperature) play an important role in these systems [1,7,24-31]. For example, both the repulsive and attractive components of the interactions have been found to be significantly modified in the presence of an electrolyte [24,25,32]. As a consequence of this, the concentration of protein at which nanoparticle-protein systems transform from one phase to two phase has been dramatically suppressed [25]. The decrease in the electrostatic repulsion for lysozyme and increase in depletion attraction for BSA are found to be mainly responsible for the differences in the phase behavior. The size of the nanoparticles is another parameter which plays a vital role in the nanoparticle-protein interactions $[13,14,33-36]$. Tuning the size of the nanoparticles not only changes the curvature but also the surface area of the nanoparticles for protein interaction. In the case of protein adsorbing on the nanoparticles, the competition of decreasing curvature (favoring adsorption) and decreasing overall surface area (disfavoring adsorption) with increasing the nanoparticle size controls the adsorption behavior of protein on the nanoparticles [37,38]. On the other hand, for nonadsorbing proteins, the excluded volume increases and the number density of the nanoparticles decreases with increasing the size of the nanoparticles. Both of these effects lead to the enhancement of depletion interaction [39-41]. In the present paper, we have examined the interactions and resultant structures of nanoparticle-protein systems for both the adsorbing and nonabsorbing proteins for different sized nanoparticles.

Herein, we have studied the interactions of different sized anionic silica nanoparticles with two model proteins-cationic lysozyme and anionic BSA. The adsorption isotherm and phase behavior as well as interactions and resultant structure in these systems have been examined by a combination of spectroscopy and scattering techniques. UV-vis spectroscopy has been used for determining the adsorption isotherm and phase behavior. The composition of a phase in this technique is determined through the differences in the absorption spectra of different components [42]. The scattering techniquesdynamic light scattering (DLS) and small-angle neutron scattering (SANS) - are most commonly used for structural and interaction details in such systems [29,43]. DLS measures the autocorrelation function of scattered light intensity from which the diffusion coefficient of the particles and hence the apparent particle size can be calculated [44]. SANS provides information about both the structure and interaction through form factor and structure factor of the scattering intensity, respectively [45]. The nanoparticle size-dependent effects are explained in terms of the modifications in their interactions with proteins and resultant structures. The interactions have been modeled by the two-Yukawa (2Y) potential to take account of competitive attractive and repulsive components in the system [46].

\section{EXPERIMENTS}

The electrostatically stabilized colloidal suspensions of different sized spherical silica nanoparticles (Ludox SM30, HS40, and TM40) and lyophilized powder proteins (hen egg lysozyme and BSA) were purchased from Sigma-Aldrich. Different compositions of nanoparticle-protein samples were prepared in $20 \mathrm{~m} M$ phosphate buffer at $p \mathrm{H} 7$ keeping a fixed concentration of silica nanoparticles $(1 \mathrm{wt} \%)$ and varying the concentration of proteins in the range $0-5 \mathrm{wt} \%$. The $p \mathrm{H}$ value for different samples was measured and found to be $7.0 \pm 0.2$. The adsorption isotherm of nanoparticle-protein interaction was studied using an ND 1000 nanodrop spectrophotometer. The instrument has a pulsed xenon flash lamp as a source with spectrum range from 2200 to $7500 \AA$ and data were measured by CCD arrays. DLS measurements were performed on a nanoparticle size analyzer, SZ-100 (HORIBA, Japan). This instrument has an incident laser light of wavelength $5320 \AA$ and the data were collected at scattering angle $173^{\circ}$ using a photomultiplier tube (PMT) detector. Small-angle neutron scattering experiments were performed on the SANS-I instrument at the Swiss spallation neutron source, SINQ, Paul Scherrer Institut, Switzerland [47]. The mean wavelength $(\lambda)$ of the incident neutron beam was $6 \AA$ with the wavelength resolution of approximately $10 \%$. A large $\left(96 \times 96 \mathrm{~cm}^{2}\right)$ $\mathrm{He}^{3}$ area detector is used to collect the scattered neutrons. The experiments were performed at two sample-to-detector distances of 2 and $8 \mathrm{~m}$ to cover the data over a scattering vector [ $Q=4 \pi \sin (\theta / 2) / \lambda$, where $\theta$ is the scattering angle] range of $0.006-0.25 \AA^{-1}$. The data were corrected and normalized to absolute scale using standard procedure. The temperature was kept constant at $30^{\circ} \mathrm{C}$ during all the measurements.

\section{DATA ANALYSIS}

\section{A. Small-angle neutron scattering}

In SANS, coherent differential scattering cross section per unit volume $(d \Sigma / d \Omega)$ is measured as a function of scattering vector, $Q$, and for a system of interacting monodisperse particles it can be expressed as $[22,45,48]$

$$
\frac{d \Sigma}{d \Omega}(Q)=\Phi V\left(\rho_{p}-\rho_{s}\right)^{2} P(Q) S(Q)+B,
$$

where $V$ is the volume of the individual particle and $\Phi$ is their volume fraction. $\rho_{p}$ and $\rho_{s}$ are scattering length densities of particles and solvent, respectively. $P(Q)$ is the orientational average of the square of form factor $F(Q)$ and is called the intraparticle structure factor. It is a measure of spatial correlation of scattering within the particle and thus gives information about the shape and size of the particle. $S(Q)$ is the interparticle structure factor and it describes the interference (correlation) of scattering from different particles. It contains the information about the interactions between particles and their spatial arrangements. $B$ is a constant term representing incoherent background.

The manifestation of interactions between particles in $S(Q)$ can be accounted using the two-Yukawa potential (2Y) for attractive and repulsive components under the mean spherical approximation [46]. The $2 \mathrm{Y}$ potential having four dimensionless parameters $\left(K_{1}, K_{2}, Z_{1}\right.$, and $\left.Z_{2}\right)$ is expressed by

$$
\begin{aligned}
\frac{V(r)}{k_{B} T} & =\infty(0<r<\sigma) \\
& =\frac{V_{2 Y}}{k_{B} T}(r>\sigma),
\end{aligned}
$$



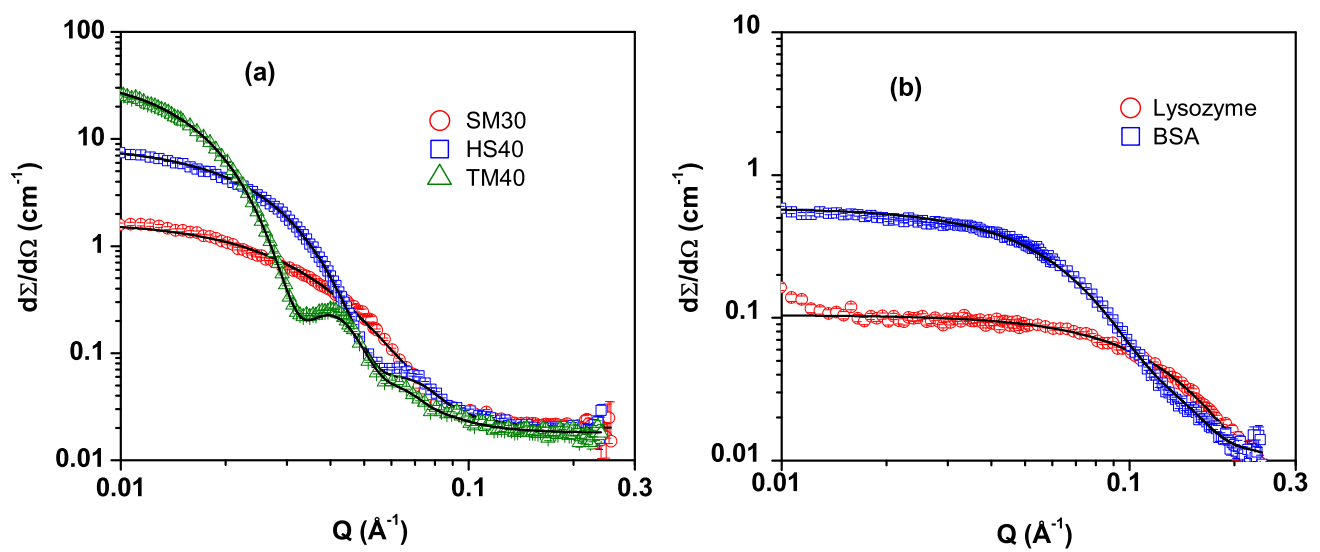

FIG. 1. SANS data of $1 \mathrm{wt} \%$ (a) silica nanoparticles (SM30, HS40, and TM40) and (b) proteins (lysozyme and BSA) in $\mathrm{D}_{2} \mathrm{O}$ at $p H \approx 7$.

where

$$
\frac{V_{2 Y}(r)}{k_{B} T}=-K_{1} \frac{\exp \left[-Z_{1}(r / \sigma-1)\right]}{r / \sigma}+K_{2} \frac{\exp \left[-Z_{2}(r / \sigma-1)\right]}{r / \sigma},
$$

where $k_{B}$ is Boltzmann's constant, $T$ is the absolute temperature, $r$ is the interparticle distance, and $\sigma$ is the hard sphere diameter of the particle.

\section{B. Dynamic light scattering}

DLS measurement yields the intensity autocorrelation function $\left[g^{(2)}(\tau)\right]$ of scattered light. For an ergodic system it is related to the field autocorrelation function $\left[g^{(1)}(\tau)\right]$ by the Siegert relation as $[44,49]$

$$
g^{(2)}(\tau)=\beta\left[g^{(1)}(\tau)\right]^{2}+1,
$$

where $\tau$ is the delay time in the correlation function and $\beta$ is an experimental constant called the spatial coherence factor whose magnitude depends on the optical geometry $(0<\beta<1)$.

In case of a monodisperse system of particles, $g^{(1)}(\tau)$ decays exponentially and for a polydisperse system it can be written as [50]

$$
g^{(1)}(\tau)=\int_{0}^{\infty} G(\Gamma) \exp (-\Gamma \tau) d \Gamma,
$$

where $\Gamma\left(=D Q^{2}\right)$ is the decay constant for a given size with the diffusion coefficients, $D$, of the particles and the magnitude of wave vector, $Q$, and $G(\Gamma)$ is the weight factor in the decay constant distribution. In the case of monomodal distribution, cumulant analysis can be used to calculate the average decay rate and the polydispersity. In this analysis, for a narrow distribution of particle size, Eq. (5) can be written as [50]

$$
g^{(1)}(\tau)=\exp \left[-\bar{\Gamma} \tau+\frac{\mu_{2} \tau^{2}}{2}\right],
$$

where $\bar{\Gamma}$ is the average decay constant and $\mu_{2}$ is the variance. The ratio of variance to the square of the average decay constant is called the polydispersity index (PI). From the average decay constant the average diffusion coefficients and hence the hydrodynamic size by the Stokes-Einstein relation can be calculated.

\section{RESULTS AND DISCUSSION}

Figure 1 shows the SANS data of $1 \mathrm{wt} \%$ of three different sized silica nanoparticles (SM30, HS40, and TM40) and proteins (lysozyme and BSA) prepared in $\mathrm{D}_{2} \mathrm{O}$ at $p \mathrm{H}$ 7. The features of SANS data are observed to be significantly different for the nanoparticles and proteins. This variation in SANS profiles can be attributed to differences in size and shape as well as contrast of the constituent with respect to the solvent [45]. At low concentration $(1 \mathrm{wt} \%)$ and in the absence of any correlation peak, these systems can be treated as noninteracting dilute systems; i.e., $S(Q) \sim 1$ [22]. This has been, in fact, confirmed by scaling of the SANS data of lower concentration (say, $0.5 \mathrm{wt} \%$ ) to $1 \mathrm{wt} \%$. Thereby, the scattering is mainly governed by the form factor of the scatterers. The magnitude of scattering from such dilute systems increases with the volume of individual particles and the width of the scattering profile is reciprocal to the size of the particles [Eq. (1)]. This suggests that qualitatively the sizes of the nanoparticles are larger than the proteins used. It is also clear that the TM40 nanoparticles are the largest among the three nanoparticles and BSA is larger than the lysozyme protein. The SANS data have been fitted for the form factor of polydisperse spheres for the nanoparticles and different ellipsoids for the proteins. The values of fitted parameters are given in Table I. The mean radii of SM30, HS40, and TM40 are found to be 5.0, 8.8, and $13.8 \mathrm{~nm}$ with polydispersity $0.25,0.16$, and 0.13 , respectively. A prolate ellipsoid having semimajor axis $2.40 \mathrm{~nm}$ and semiminor axis $1.35 \mathrm{~nm}$ for lysozyme, and an oblate ellipsoid with semimajor axis $4.20 \mathrm{~nm}$ and semiminor axis $1.50 \mathrm{~nm}$ for BSA have been fitted. These structural parameters of both the nanoparticles and proteins are found to be in agreement with earlier reported values [24-26,51]. The visibility of form factor oscillations at higher $Q$ region [Fig. 1(a)] depends on the polydispersity of the particles and their smearing increases with the polydispersity. The relatively poor resolution in the case of SANS also smears these oscillations as reflected in the protein data [Fig. 1(b)]. The main reason for poor $Q$ resolution $(\Delta Q)$ arises from the contribution of wavelength spread $(\Delta \lambda / \lambda)$, where $\Delta Q$ 
TABLE I. Fitted parameters of $1 \mathrm{wt} \%$ of silica nanoparticles (SM30, HS40, and TM40) and $1 \mathrm{wt} \%$ proteins (lysozyme and BSA) in $\mathrm{D}_{2} \mathrm{O}$ at $p H \approx 7$.

\begin{tabular}{|c|c|c|c|c|c|c|}
\hline \multicolumn{7}{|c|}{ (a) Silica nanoparticles } \\
\hline $\begin{array}{c}\text { Nanoparticle } \\
\text { system }\end{array}$ & $\begin{array}{l}\text { Mean radius } \\
R_{\mathrm{m}}(\mathrm{nm})\end{array}$ & Polydispersity $\sigma$ & $\begin{array}{c}\text { Surface } \\
\text { area per } \\
\text { particle } \\
\left(\mathrm{nm}^{2}\right)\end{array}$ & $\begin{array}{c}\text { Number } \\
\text { density } \\
n_{\mathrm{NP}}\left(\mathrm{m}^{-3}\right)\end{array}$ & $\begin{array}{c}\text { Specific } \\
\text { surface } \\
\text { area } \\
\left(\mathrm{m}^{2} / \mathrm{g}\right)\end{array}$ & $\begin{array}{l}\text { Curvature } \\
\left(\mathrm{nm}^{-1}\right)\end{array}$ \\
\hline SM30 & 5.00 & 0.25 & $3.1 \times 10^{2}$ & $8.59 \times 10^{21}$ & 272 & 0.20 \\
\hline HS40 & 8.80 & 0.16 & $9.7 \times 10^{2}$ & $1.57 \times 10^{21}$ & 155 & 0.11 \\
\hline TM40 & 13.80 & 0.13 & $2.4 \times 10^{3}$ & $4.08 \times 10^{20}$ & 99 & 0.07 \\
\hline \multicolumn{7}{|c|}{ (b) Proteins } \\
\hline Protein system & Shape & Semimajor axis $a(\mathrm{~nm})$ & $\begin{array}{l}\text { Semiminor } \\
\text { axis } b \\
(\mathrm{~nm})\end{array}$ & $\begin{array}{c}\text { Equivalent } \\
\text { radius } \\
R_{\mathrm{e}}(\mathrm{nm})\end{array}$ & $\begin{array}{c}\text { Surface } \\
\text { area per } \\
\text { molecule } \\
\left(\mathrm{nm}^{2}\right)\end{array}$ & $\begin{array}{c}\text { Number } \\
\text { density } n_{\mathrm{P}} \\
\left(\mathrm{m}^{-3}\right)\end{array}$ \\
\hline Lysozyme & Prolate ellipsoidal & 2.40 & 1.35 & 1.63 & 35 & $4.09 \times 10^{23}$ \\
\hline BSA & Oblate ellipsoidal & 4.20 & 1.50 & 2.98 & 130 & $9.07 \times 10^{22}$ \\
\hline
\end{tabular}

increases linearly with $Q$ [52,53]. The scattering buildup at low $Q$ for lysozyme could be from the existence of some permanent aggregates in the system [54]. The calculated values of the surface area per particle, and number density of the nanoparticles and protein systems, as well as the specific surface area and curvature of the nanoparticles are also given in Table I. There is an increase in surface area per nanoparticles, about one order, and a decrease in number density by two orders among the nanoparticles. The overall area for the nanoparticles (specific surface area) and curvature decreases with the increase in size of the nanoparticles. The significant difference in size of the nanoparticles and proteins allows a high enough number of proteins to interact with the nanoparticles.

The adsorption isotherms of lysozyme and BSA proteins on three different sized silica nanoparticles as obtained by UV-vis spectroscopy are presented in Fig. 2. The measurements were carried out for a fixed concentration (1 wt \%) of silica nanoparticles with a varying concentration of lysozyme and BSA proteins. The adsorption isotherms are determined by separating protein adsorbed nanoparticles from the free protein, if any. The separation is carried out by centrifuging samples at
$18000 \mathrm{~g}$ for $30 \mathrm{~min}$. The absorbance spectra, which show a peak at about $280 \mathrm{~nm}$ for both proteins, are used to determine the proteins' concentration in the supernatant $[13,15,16]$. The amounts of adsorbed proteins are calculated from the difference between initial (total) and supernatant concentration of proteins. The data in Fig. 2 show the amount of adsorbed protein on silica nanoparticles vs total protein in the system. The adsorption isotherms irrespective of the nanoparticle size show similar features. The adsorption of lysozyme increases with concentration and follows typical exponential growth behavior [Fig. 2(a)]. On the other hand, BSA protein does not show any adsorption [Fig. 2(b)]. The electrostatic repulsion between similarly charged nanoparticles and BSA protein over their site-specific attraction seems to prevent the adsorption of BSA protein on the nanoparticles. The data of lysozyme protein are fitted with equation $A=A_{0}\left[1-\exp ^{-K C}\right]$, where $A$ is the adsorbed protein, $A_{0}$ is the saturation value, $K$ is the adsorption coefficient, and $C$ is the total lysozyme concentration. The fitted parameters are given in Table II. The adsorption coefficient increases whereas saturation value decreases with increasing the size of the nanoparticles. The higher value of saturation in the case of smaller size of the
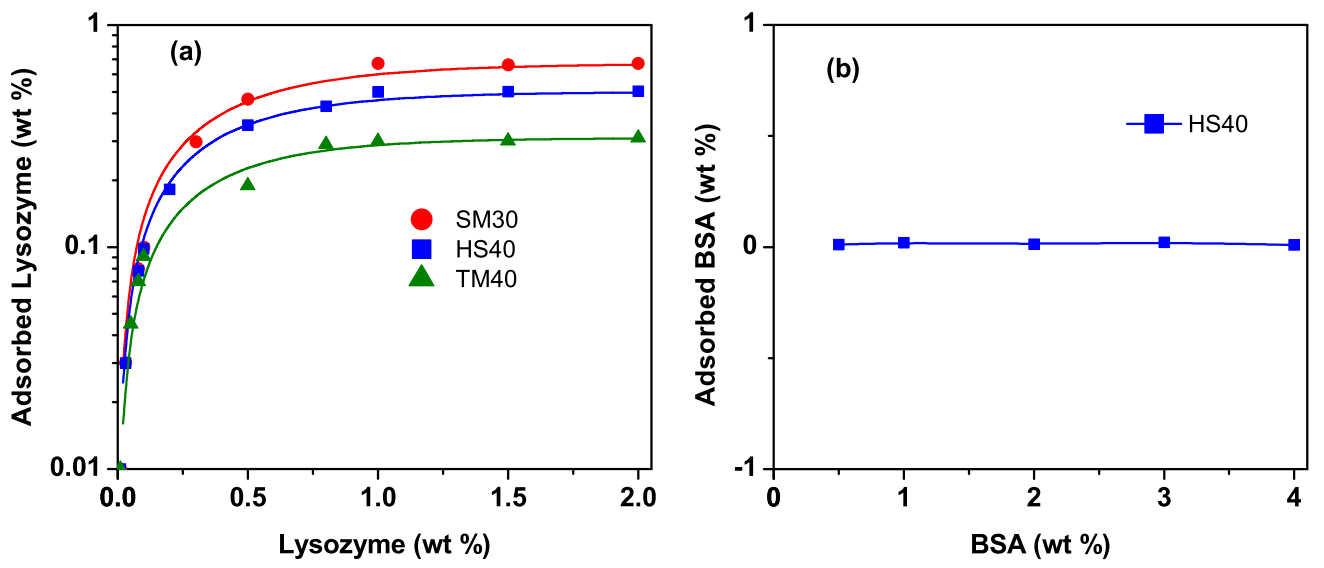

FIG. 2. Adsorption isotherm of (a) lysozyme and (b) BSA proteins on $1 \mathrm{wt} \%$ silica nanoparticles at $p H \approx 7$. Lysozyme shows an exponential growth behavior whereas BSA does not adsorb, irrespective of the nanoparticle size. 
TABLE II. Fitted parameters of adsorption curves of lysozyme protein on $1 \mathrm{wt} \%$, different sized silica nanoparticles at $p H \approx 7$. $N_{\mathrm{p}}, N_{\mathrm{s}}$, and $\mathrm{PF}$ are the number of adsorbed lysozyme molecules per nanoparticle, surface density of adsorbed lysozyme, and packing fraction, respectively, at saturation value.

\begin{tabular}{lccccc}
\hline \hline $\begin{array}{c}\text { Nanoparticle } \\
\text { system }\end{array}$ & $\begin{array}{c}\text { Saturation } \\
\text { value } A_{0} \\
(\text { wt } \%)\end{array}$ & $\begin{array}{c}\text { Adsorption } \\
\text { coefficient } \\
K(1 / \mathrm{wt} \%)\end{array}$ & $N_{\mathrm{P}}$ & $N_{\mathrm{s}}\left(\mathrm{m}^{-2}\right)$ & $\mathrm{PF}$ \\
\hline SM30 & 0.67 & 2.25 & 32 & $10.2 \times 10^{16}$ & 0.48 \\
HS40 & 0.50 & 2.49 & 130 & $13.4 \times 10^{16}$ & 0.79 \\
TM40 & 0.31 & 2.63 & 310 & $13.5 \times 10^{16}$ & 0.86 \\
\hline \hline
\end{tabular}

nanoparticles (SM30) can be attributed to the larger total surface area available for protein adsorption [Table I]. The contact area of the protein is expected to increase with the decrease in the nanoparticle curvature (increasing nanoparticle size) and hence the adsorption coefficient increases with the nanoparticle size. From the adsorption data, the number of adsorbed lysozyme molecules per nanoparticles, surface number density, and packing fraction of adsorbed lysozyme have also been calculated [Table II]. The number of adsorbed proteins per nanoparticle as expected increases with the size of the nanoparticles. The maximum adsorption of protein on nanoparticles $\left(A_{0}\right)$ concomitant with the total surface area of the nanoparticles increases with the decrease in the size of the nanoparticles [Table II]. This increase (about 2 times) is not scaled solely by change in the surface area (about 3 times) due to the important curvature effect, as reflected in the significant reduction in the surface number density and packing fraction of adsorbed protein on the nanoparticles with decrease in the size of the nanoparticles $[13,38]$.

The adsorption of lysozyme leads to the bridging aggregation of the nanoparticle and thereby nanoparticle-protein system transform from one phase (clear) to two phase (turbid) $[15,16]$. Interestingly, similar behavior is also observed for the nonadsorbing BSA for much higher concentration than lysozyme. The phase behavior is examined by measuring the transmission of light $(6000 \AA)$ through silica nanoparticles $(1 \mathrm{wt} \%)$ as a function of protein concentration. The sizedependent phase behavior of the nanoparticles with proteins is shown in Fig. 3. The two-phase nanoparticle-protein system is identified by a sudden decrease in the transmission of light. The phase behavior in Fig. 3 shows similar features irrespective of protein type and size of the nanoparticles. However, the aggregation commences at lower concentration for the larger sized nanoparticles for both proteins (insets of Fig. 3). In the case of lysozyme, they adsorb on the surface of the oppositely charged nanoparticles neutralizing the charge and thereby causing protein-mediated aggregation of the nanoparticles (formation of two-phase system) [15,16] The critical protein concentration (CPC) to induce phase transformation [inset of Fig. 3(a)] is governed by the charge neutralization through the competition of surface area effect (the larger the size, the higher will be the protein required) and particle number density effect (the higher the size, the lower will be the protein required) as decided by the number ratio of protein to nanoparticle $[15,24]$. The decrease in CPC with increase in size suggests the dominance of the number density effect over the surface area effect. For similarly charged BSA, the phase transformation arises from their nonadsorption leading to the depletion-induced aggregation of the nanoparticles [22,25]. The CPC [inset of Fig. 3(b)] in this case is lowered with the increase in the size of the nanoparticles because of enhancement of the excluded volume effect of individual nanoparticles (the higher the size, the higher will be the excluded volume) as well as the number density effect (the higher the size, the higher will be the protein molecules per nanoparticle). The evolution of interaction and structure in these systems has been studied by DLS and SANS.

The DLS autocorrelation function of one of the nanoparticle systems (HS40) at different concentrations of lysozyme in their phase behavior [Fig. 3] are shown in Fig. 4(a). The autocorrelation function is systematically broadened with the increase in the concentration of lysozyme. The broadening of autocorrelation function suggests a decrease in the diffusion coefficient of the particles in the system. This can arise due to the evolution of attractive interaction between the nanoparticles and/or the formation of the nanoparticle aggregates mediated by the lysozyme. The calculated size distributions of the nanoparticles with varying lysozyme concentration using Eq. (6) employing CONTIN analysis are given in Fig. 4(b) [50]. The size distributions of the nanoparticles show interesting features where the mean size of the distribution shifts to larger values with increase in lysozyme
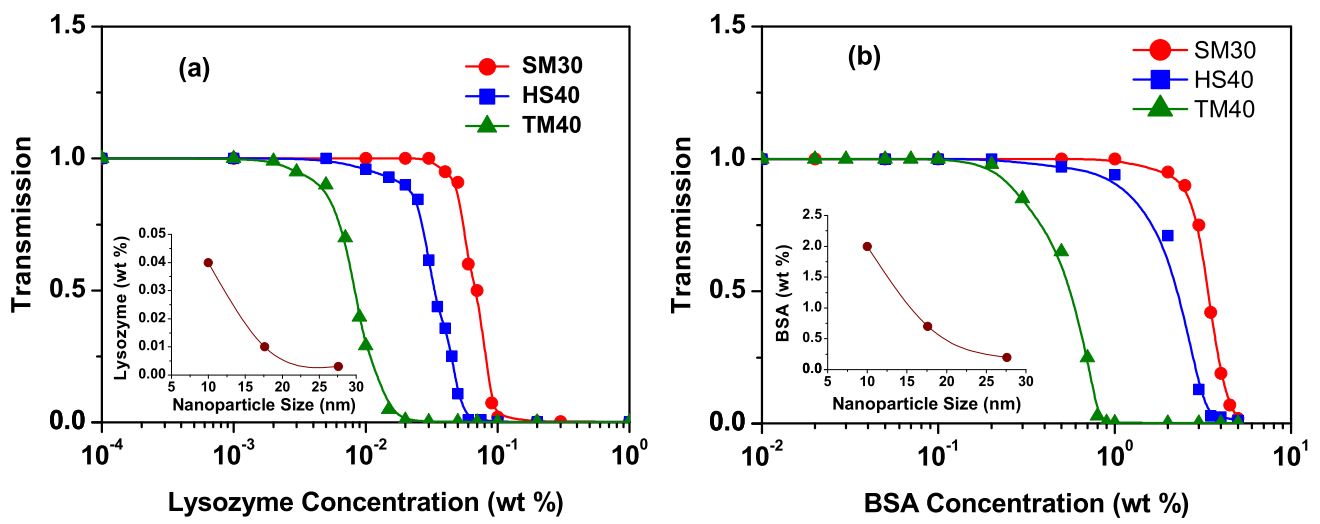

FIG. 3. Transmission of light in $1 \mathrm{wt} \%$ of silica nanoparticles (SM30, HS40, and TM40) with varying concentrations of (a) lysozyme and (b) BSA proteins at $p H \approx 7$. Insets show the size-dependent variation of critical protein concentration for the two-phase system. 

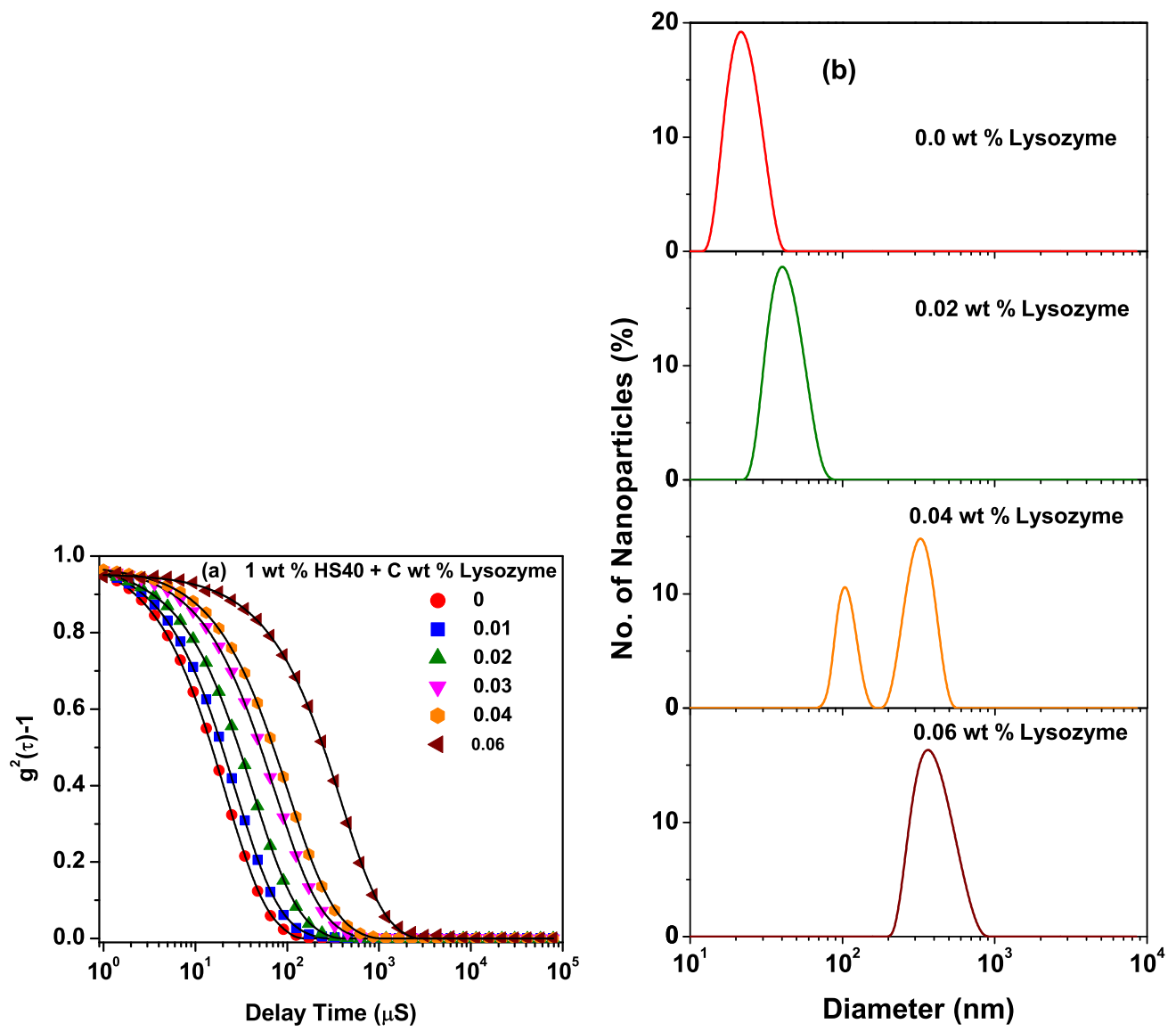

FIG. 4. DLS data of $1 \mathrm{wt} \%$ HS40 silica nanoparticles with varying concentration of lysozyme at $p H \approx 7$. (a) Autocorrelation function and (b) particle size distribution.

concentration. Moreover, there exists a concentration range of lysozyme for which the size distribution is bimodal. Based on these observations, phase behavior can be divided into three regions. In region I, the increase in effective size of the nanoparticles corresponds to buildup of attractive interaction prior to the two-phase formation. Region II represents the coexistence of one-phase (nanoparticles undergoing attractive interaction) and two-phase (nanoparticles aggregate) systems as characterized by bimodal distribution. The polydispersity is believed to be responsible for the coexistence of the one-phase system with the two-phase system. Region III belongs to the two-phase system having mostly nanoparticle aggregates. Nanoparticles, irrespective of their size, show similar features of evolution of interaction and structure in respective phase behavior. However, the concentration of protein needed to get the required changes is lowered in favor of large sized nanoparticles because of the interplay of surface area, number density, and curvature effects in the system.

It has been seen that in spite of different interactions (adsorption isotherms in Fig. 2) of lysozyme and BSA proteins with silica nanoparticles, both proteins show similar phase behavior [Fig. 3]. The attractive interaction responsible for the aggregation of the nanoparticles in the presence of BSA protein is induced by depletion from the nonadsorption of protein. Figure 5 shows the DLS data of the nanoparticle system (HS40) as a function of BSA concentration. The inset of Fig. 5(a) shows the DLS autocorrelation function in the low concentration region of BSA. In this case, there are no significant changes in autocorrelation function observed. This supports the fact that BSA protein is nonadsorbing to the nanoparticles. The changes in autocorrelation function at higher BSA concentrations [Fig. 5(a)] are due to evolution of the depletion interaction and resultant structures. The strength of the depletion interaction is known to increase with the depletant (BSA) concentration [55-57]. Similar to the nanoparticlelysozyme system, the evolution of interaction and structure are manifested in DLS data and can be divided into three regions. In region $\mathrm{I}$, the depletion-induced attractive interaction slows down the diffusion of the individual nanoparticles and hence increases the apparent size of the nanoparticles. The further increase in depletion interaction with BSA concentration leads to aggregation of the nanoparticles in region II. This region is characterized by a bimodal distribution of a part of the unaggregated nanoparticles coexisting with the aggregated nanoparticles. There are mostly the nanoparticle aggregates in region III at higher BSA concentrations. Similar behavior has also been observed for the other two nanoparticles (SM30 and TM40) in the presence of BSA proteins and the results are consistent with the corresponding phase behavior.

We have observed in DLS data that the phase behavior of nanoparticle-protein systems can be interpreted in terms of evolution of attractive interaction and/or aggregation. However, DLS has limitations in separating contributions of interaction and structure, both of which can influence 

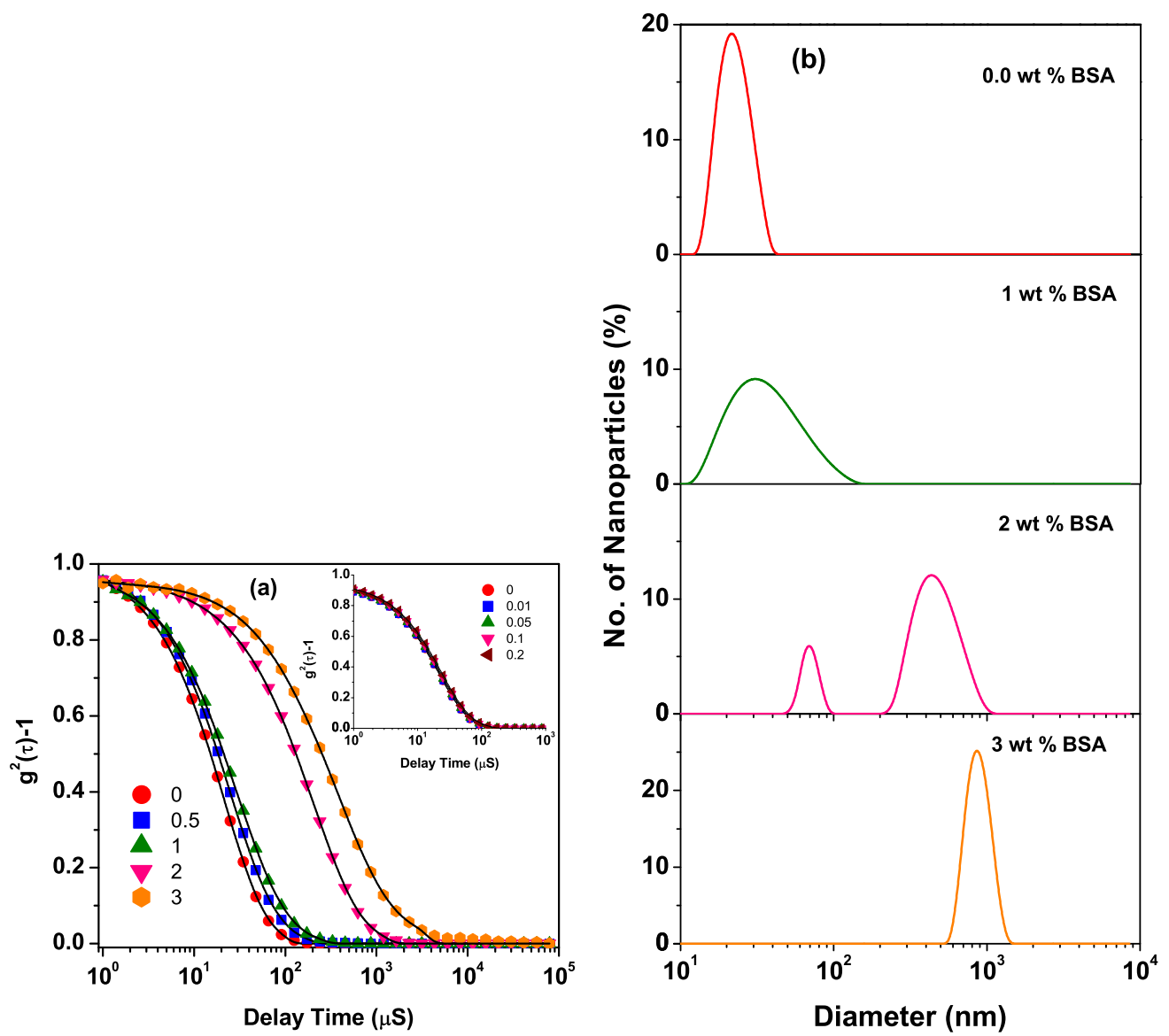

FIG. 5. DLS data of $1 \mathrm{wt} \%$ HS40 silica nanoparticles with varying concentration of BSA at $p H \approx 7$. (a) Autocorrelation function and (b) particle size distribution. Inset in (a) shows the autocorrelation function at low BSA concentrations.

the data the same way. SANS can measure structure and interaction separately through the form factor and structure factor of the scattering intensity, respectively. The SANS data of HS40 silica nanoparticles with lysozyme in their phase behavior [Fig. 3] are shown in Fig. 6. Significant changes in scattering profiles have been found as a function of lysozyme concentration. Based on the $Q$-dependent features, data at different protein concentrations can be divided into two regions, low $Q\left(<0.03 \AA^{-1}\right)$ and high $Q\left(>0.03 \AA^{-1}\right)$. There is systematic buildup in scattering intensity in the low $Q$ region whereas no significant changes have been found in the high $Q$ region. The scattering from lysozyme is about two orders less than the nanoparticles and hence the rise of scattering in low $Q$ from the nanoparticle-lysozyme system cannot be additive from the individual components. The changes in scattering in low $Q$ are expected due to the evolution of interaction and/or structure in the nanoparticles in the presence of protein as observed in DLS data [25]. On the other hand, the data in high $Q$ are governed by the additive form factor of the nanoparticles and protein [22]. The features of the buildup in scattering intensity in low $Q$ are similar to that of attractive interaction in the system. The scattering intensity at $S(Q=0)$ is inversely related to the osmotic pressure of the system. This results in a very high value of $S(Q=0)$ for attractive interaction and $S(Q)$ diverges in the low $Q$ region. The SANS data are therefore fitted with the $S(Q)$ of attractive interaction between nanoparticles as induced by the presence of protein. The fitted parameters are given in Table III(a). Further, SANS data can be divided into three groups depending upon the concentration of lysozyme corresponding to different regions of phase behavior

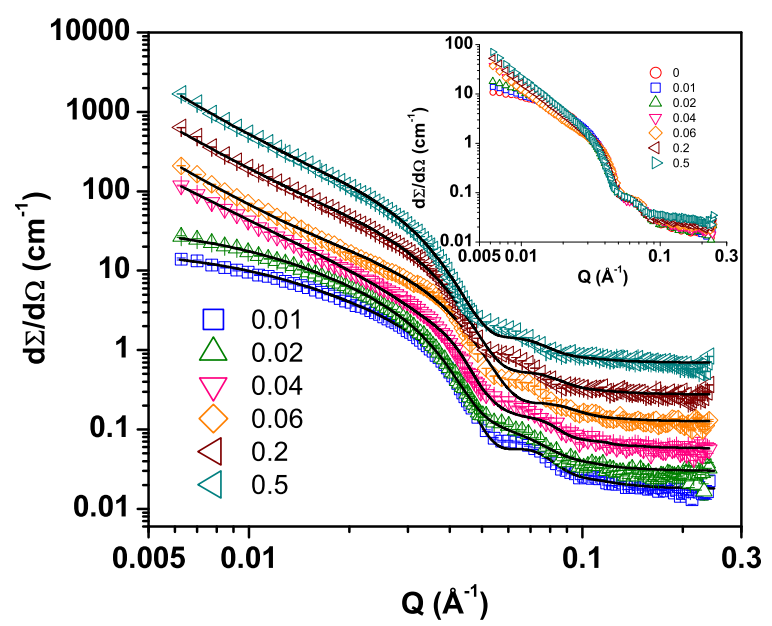

FIG. 6. SANS data of $1 \mathrm{wt} \%$ HS40 silica nanoparticles with varying concentration of lysozyme protein at $p H \approx 7$. Data have been scaled for clarity whereas the inset shows the same set of data without scaling. 
TABLE III. Fitted parameters of silica nanoparticles with lysozyme protein at $p H \approx 7$.

\begin{tabular}{|c|c|c|c|c|c|}
\hline $\begin{array}{c}\text { Lysozyme } \\
\text { concentration } C \\
(\mathrm{wt} \%)\end{array}$ & $\begin{array}{c}\text { Fractal } \\
\text { dimension } \\
\left(D_{\mathrm{m}}\right)\end{array}$ & $\begin{array}{c}\text { Building } \\
\text { block radius } \\
R_{\mathrm{b}}(\AA)\end{array}$ & $K_{1}$ & $Z_{1}$ & $\begin{array}{c}\text { Fraction of } \\
\text { unaggregated } \\
\text { nanoparticles } \phi_{\text {unp }} \\
(\%)\end{array}$ \\
\hline 0.01 & & & 18 & 9.0 & 100 \\
\hline 0.02 & & & 20 & 9.0 & 100 \\
\hline 0.04 & 2.4 & 90.0 & 24 & 9.5 & 55 \\
\hline 0.06 & 2.4 & 92.0 & 28 & 11.0 & 35 \\
\hline 0.2 & 2.5 & 93.2 & 40 & 14.5 & 10 \\
\hline 0.5 & 2.5 & 94.2 & & & 0 \\
\hline
\end{tabular}

(b) $1 \mathrm{wt} \%$, different sized silica nanoparticles (SM30, HS40, and TM40) with lysozyme protein at their respective CPC values.

\begin{tabular}{lccccc}
\hline $\begin{array}{c}\text { Nanoparticle } \\
\text { system }\end{array}$ & $\begin{array}{c}\text { Lysozyme } \\
\text { concentration } \\
\text { (wt \%) }\end{array}$ & $K_{1}$ & $Z_{1}$ & $K_{2}$ & $Z_{2}$ \\
\hline SM30 & 0.04 & 7 & 5 & 4.5 & 3.5 \\
HS40 & 0.01 & 18 & 9 & 9 & 7 \\
TM40 & 0.003 & 40 & 14 & 14 & 11 \\
\hline \hline
\end{tabular}

[Fig. 3(a)]. The first group of SANS data corresponds to the phase behavior where the nanoparticle-protein system remains in one phase. Data have been fitted with the $2 \mathrm{Y}$ potential taking account of both the attractive as well as repulsive parts of the parameters $\left(K_{1}, K_{2}, Z_{1}, Z_{2}\right)$. Here, $K_{1}$ and $Z_{1}$ are the fitting parameters of the attractive potential to give the strength (proportional to $K_{1}$ ) and range (proportional to $1 / Z_{1}$ ), respectively. On the other hand, $K_{2}$ and $Z_{2}$ are the fitting parameters of the repulsive potential which provide the strength (related to effective charge) and range (related to ionic strength), respectively [46]. The parameters of repulsive potential have been determined from the pure concentrated solution of the nanoparticles and kept fixed during the data analysis [22]. It is found that unlike the long-range repulsion between charged nanoparticles the protein-mediated attraction is short range [24,25]. The range of attraction is found to be of the order of the size of the protein over which it mediates between the nanoparticles. The second group of SANS data belongs to phase behavior where the one-phase region coexists with the two-phase region. For this group of data, the bimodal distribution has been observed in DLS data. The SANS data in the group are fitted with the two contributions from the aggregates of the nanoparticles and the remaining fraction of individual nanoparticles undergoing attractive interaction. The fraction of the nanoparticle aggregates increases with the concentration of lysozyme. Data of the third group have been fitted only by nanoparticle aggregates. The morphology of the nanoparticle aggregates is found to be mass fractal, having a fractal dimension about 2.4. The calculated value of the fractal dimension (2.4) also suggests that the aggregates of the nanoparticles are formed through the diffusion limited process.

It has been observed that the CPC value strongly depends on the nanoparticle size as discussed earlier and it is significantly smaller for larger sized nanoparticles. The SANS data of different sized silica nanoparticles with lysozyme protein at close to their respective CPC values are shown in Fig. 7(a). The data in each case have been fitted with the $2 \mathrm{Y}$ potential and the values of fitted parameters are given in Table III(b). The total potential between nanoparticles and its individual components
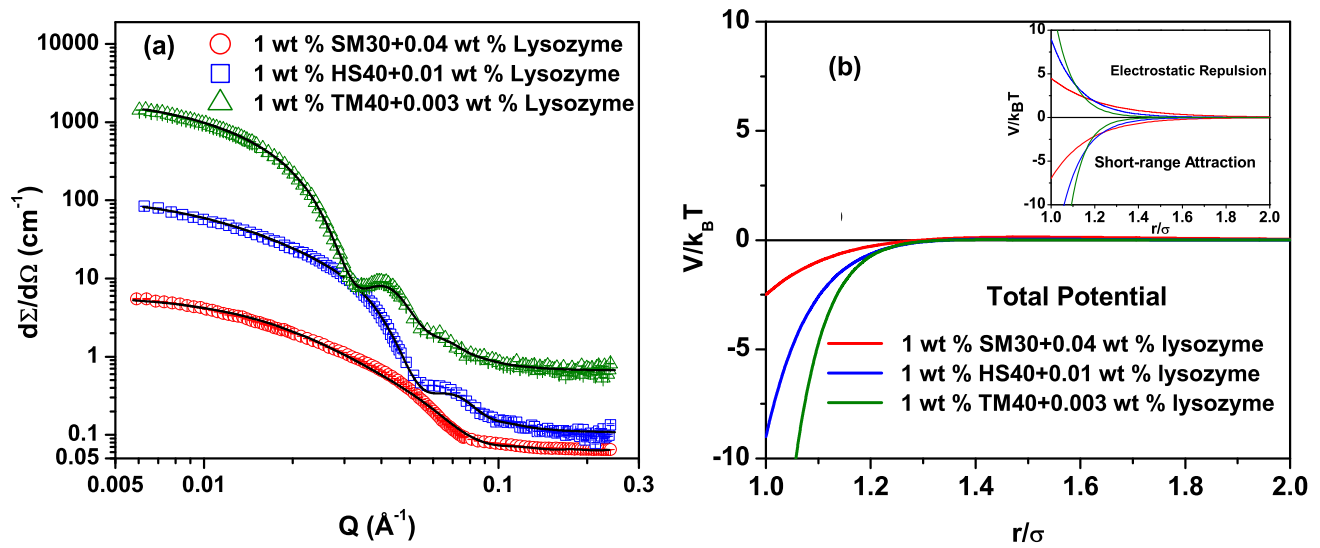

FIG. 7. (a) SANS data of $1 \mathrm{wt} \%$, three different sized silica nanoparticles with lysozyme protein at their respective CPC values, and (b) the calculated total interaction potentials along with components (inset) between nanoparticles from the fitting of SANS data at $p H \approx 7$. 
are plotted in Fig. 7(b). It has been found that the strength of repulsion $\left(K_{2}\right)$ increases with the size of the nanoparticles whereas the range $\left(\sigma / Z_{2}\right)$ remains almost the same. This is in accordance with the DLVO theory where the magnitude of the potential is proportional to the size of the nanoparticles when stabilized by the same zeta potential [58].The range depends on the ionic strength ( $20 \mathrm{~m} M$ buffer for all the system) of the solution and therefore has been found independent of the size of the nanoparticles [24,25]. Interestingly, in spite of increased repulsion between nanoparticles for larger size nanoparticles, the CPC follows the reverse trend. This happens as the attractive interaction is also enhanced with the increase in the size of the nanoparticles [Table III(b)]. It has also been observed that the CPC of all nanoparticle-protein systems corresponds to the values of adsorbed proteins per nanoparticle ( 2 for SM30, 2.6 for HS40, and 3 for TM40) which are greater than 1 . This is possibly used to reduce the repulsive interaction between nanoparticles [24,25]. The size-dependent phase behavior in Fig. 3(a) thus arises because of the dominance of protein-mediated short-range attractive interaction over the long-range repulsion between the nanoparticles. Irrespective of the size of the nanoparticles, the morphology of the nanoparticle aggregates in the two-phase system is found to be mass fractal.

The phase behavior of a nanoparticle-BSA system [Fig. 3(a)] is similar to that of a nanoparticle-lysozyme system [Fig. 3(b)], but the CPC values are much higher for BSA protein. The SANS data of HS40 silica nanoparticles with varying BSA concentration are shown in Fig. 8. Data representing different regions of phase behavior show a trend similar to that of a nanoparticle-lysozyme system. The fitted parameters are given in Table IV(a). Unlike lysozymemediated short-range attraction, BSA-induced attractive depletion interaction between charged nanoparticles is found to be long range [11,22]. The magnitude of depletion attraction increases with the concentration of BSA protein whereas the range remains almost the same [22]. At higher BSA concentrations the dominance of the depletion interaction leads to the aggregation of the nanoparticles and the system

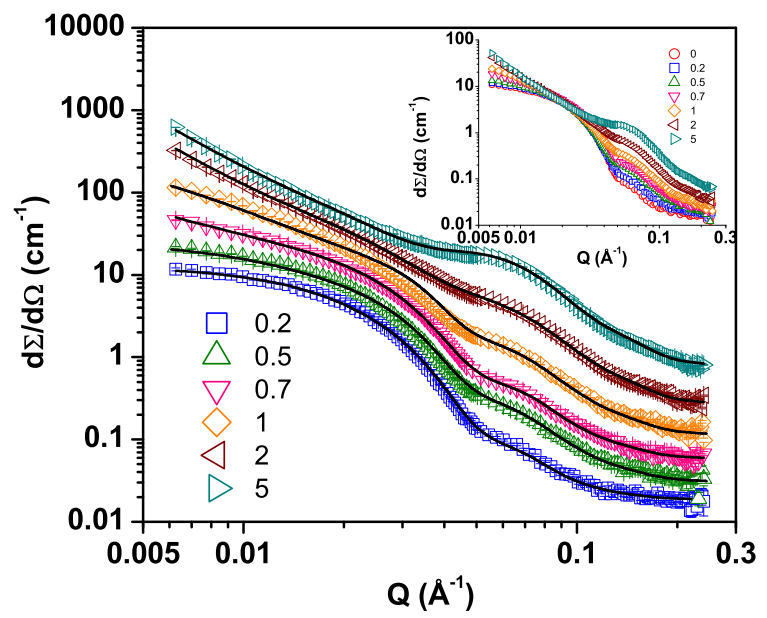

FIG. 8. SANS data of $1 \mathrm{wt} \%$ HS40 silica nanoparticles with varying concentration of BSA protein at $p H \approx 7$. Data have been scaled for clarity whereas the inset shows the same set of data without scaling.

transforms from one phase to two phase. The SANS data of different sized silica nanoparticles with BSA protein at their respective CPC values are shown in Fig. 9(a). The data have been fitted with the $2 \mathrm{Y}$ potential and the values of the fitted parameters are given in Table IV(b). The calculated resultant interaction potentials and their individual components are plotted in Fig. 9(b). Both the strength of attraction $(K 1)$ and the range $\left(\sigma / Z_{1}\right)$ of depletion interaction are found to be much larger than that of repulsion. The strength of attraction increases whereas the range remains almost the same with the increase in the size of the nanoparticles. The excluded volume effect is enhanced with increasing nanoparticle size leading to increase in the depletion interaction. It is observed that the total interaction potential of silica nanoparticles for both proteins leading to two-phase formation is more attractive for larger sized nanoparticles. This can be understood in terms of the particles' number density effect which leads to a lesser number of nanoparticles for larger size nanoparticles than smaller ones

TABLE IV. Fitted parameters of silica nanoparticles with BSA protein at $p H \approx 7$.

\begin{tabular}{|c|c|c|c|c|c|}
\hline $\begin{array}{c}\text { BSA } \\
\text { concentration } C \\
\text { (wt } \%)\end{array}$ & $\begin{array}{c}\text { Fractal } \\
\text { dimension } \\
\left(D_{\mathrm{m}}\right)\end{array}$ & $\begin{array}{c}\text { Building } \\
\text { block radius } \\
R_{\mathrm{b}}(\AA)\end{array}$ & $K_{1}$ & $Z_{1}$ & $\begin{array}{c}\text { Fraction of } \\
\text { unaggregated } \\
\text { nanoparticles } \phi_{\text {unp }} \\
(\%)\end{array}$ \\
\hline 0.2 & & & 8.5 & 4.5 & 100 \\
\hline 0.5 & & & 10.5 & 4 & 100 \\
\hline 1 & & & 26.5 & 3 & 100 \\
\hline 2 & 2.5 & 90.0 & 30 & 3 & 60 \\
\hline 5 & 2.5 & 92.5 & & & 0 \\
\hline \multicolumn{6}{|c|}{ (b) $1 \mathrm{wt} \%$, different sized silica nanoparticles (SM30, HS40, and TM40) with BSA protein at their respective CPC values. } \\
\hline $\begin{array}{l}\text { Nanoparticle } \\
\text { system }\end{array}$ & $\begin{array}{c}\text { BSA } \\
\text { concentration } \\
(w t \%)\end{array}$ & $K_{1}$ & $Z_{1}$ & $K_{2}$ & $Z_{2}$ \\
\hline SM30 & 2 & 9 & 2.5 & 4.5 & 3.5 \\
\hline HS40 & 0.7 & 20 & 3.5 & 9 & 7 \\
\hline TM40 & 0.2 & 40 & 4.5 & 14 & 11 \\
\hline
\end{tabular}



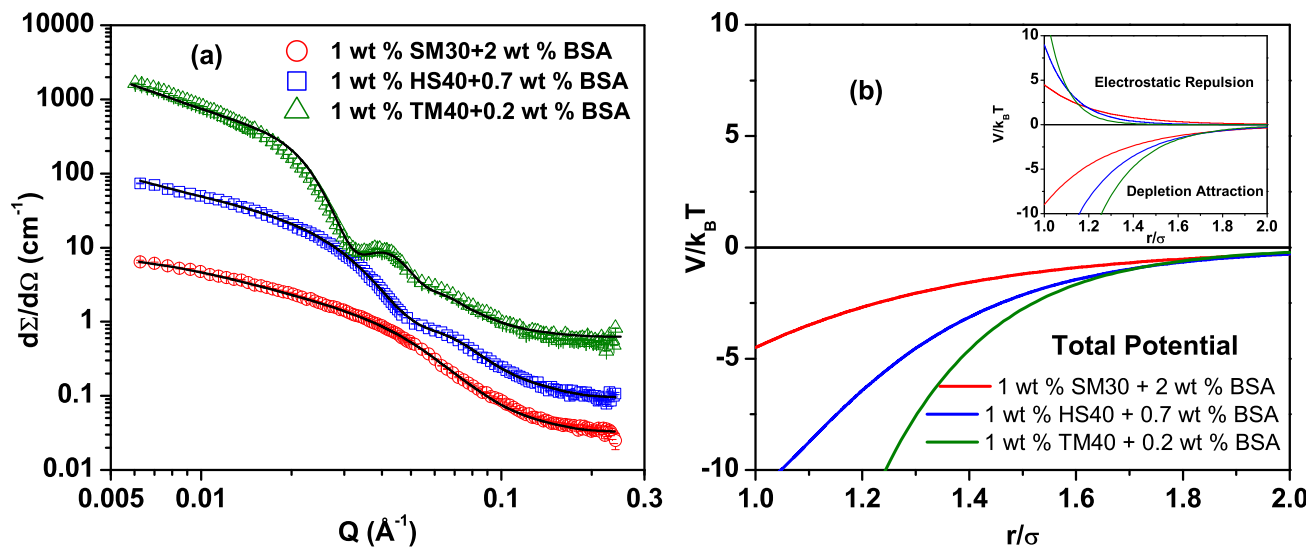

FIG. 9. (a) SANS data of $1 \mathrm{wt} \%$, three different sized silica nanoparticles with BSA protein at their respective CPC values, and (b) the calculated total interaction potentials between nanoparticles along with components (inset) from the fitting of SANS data at $p H \approx 7$.

at a constant $(1 \mathrm{wt} \%)$ concentration and therefore require a larger interaction to aggregate them [59].

\section{CONCLUSIONS}

The size-dependent interaction of silica nanoparticles with lysozyme and BSA proteins has been studied. The adsorption of lysozyme on silica nanoparticles increases with concentration and follows an exponential growth behavior. The adsorption coefficient increases whereas the saturation value decreases with increasing the size of the nanoparticles. The increase in saturation value for smaller particles is interpreted in terms of the larger total surface area available for protein adsorption. The adsorption coefficient depends on the curvature of the nanoparticles and hence favors the larger size of the nanoparticles. On the other hand, the BSA protein does not show adsorption on any size of the nanoparticles. The adsorption of lysozyme as well as nonadsorption of BSA on silica nanoparticles leads to one-phase (clear) to two-phase (turbid) transformation in the respective nanoparticle-protein system. The phase transformation is observed at lower concentration for the larger sized nanoparticles for both proteins. The decrease in CPC with increase in size for the lysozyme protein is decided by the dominance of number density effect over the surface area effect. The CPC in the case of nonadsorbing BSA protein is lowered with the increase in the size of the nanoparticles because of the enhancement of the excluded volume of an individual nanoparticle and number density effects. The hydrodynamic sizes as obtained by DLS in these systems are found to be consistent with size-dependent phase behavior of the nanoparticles with proteins. SANS suggests that the size-dependent phase behavior of silica nanoparticles for lysozyme protein arises because of the dominance of protein-mediated short-range attractive interaction over the long-range repulsion between the nanoparticles, whereas for BSA protein the excluded volume effect is enhanced with increasing nanoparticle size leading to an increase in the depletion interaction. The morphology of the nanoparticle aggregates for both proteins in the two-phase system irrespective of nanoparticle size is found to be mass fractal.
[1] I. Lynch and K. A. Dawson, Nano Today 3, 40 (2008).

[2] D. J. Bharali, I. Klejbor, E. K. Stachowiak, P. Dutta, I. Roy, N. Kaur, E. J. Bergey, P. N. Prasad, and M. K. Stachowiak, Proc. Natl. Acad. Sci. USA 102, 11539 (2005).

[3] R. A. Mcmillan, C. D. Paavola, J. Howard, S. L. Chan, N. J. Zaluzec, and J. D. Trent, Nat. Mater. 1, 247 (2002).

[4] J. Leszczynski, Nat. Nanotechnol. 5, 633 (2010).

[5] W. H. Suh, Y. H. Suh, and G. D. Stucky, Nano Today 4, 27 (2009).

[6] M. De, P. S. Ghosh, and V. M. Rotello, Adv. Mater. 20, 4225 (2008).

[7] A. E. Nel, L. Mädler, D. Velegol, T. Xia, E. M. V. Hoek, P. Somasundaran, F. Klaessig, V. Castranova, and M. Thompson, Nat. Mater. 8, 543 (2009).

[8] J. N. Israelachvili, Intermolecular and Surface Forces, revised. 3rd ed. (Academic Press, Waltham, MA, 2011).

[9] Y. Min, M. Akbulut, K. Kristiansen, Y. Golan, and J. Israelachvili, Nat. Mater. 7, 527 (2008).

[10] M. Hermansson, Colloids Surf., B 14, 105 (1999).
[11] J. C. Crocker, J. A. Matteo, A. D. Dinsmore, and A. G. Yodh, Phys. Rev. Lett. 82, 4352 (1999).

[12] S. H. Brewer, W. R. Glomm, M. C. Johnson, M. K. Knag, and S. Franzen, Langmuir 21, 9303 (2005).

[13] J. Wang, U. B. Jensen, G. V. Jensen, S. Shipovskov, V. S. Balakrishnan, D. Otzen, J. S. Pedersen, F. Besenbacher, and D. S. Sutherland, Nano Lett. 11, 4985 (2011).

[14] K. Rezwan, A. R. Studart, J. Voros, and L. J. Gauckler, J. Phys. Chem. B 109, 14469 (2005).

[15] S. Kumar, V. K. Aswal, and J. Kohlbrecher, Langmuir 27, 10167 (2011).

[16] B. Bharti, J. Meissner, and G. H. Findenegg, Langmuir 27, 9823 (2011).

[17] T. J. Su, J. R. Lu, R. K. Thomas, Z. F. Cui, and J. Penfold, J. Phys. Chem. B 102, 8100 (1998).

[18] F. Catalano, G. Alberto, P. Ivanchenko, G. Dovbeshko, and G. Martra, J. Phys. Chem. C 119, 26493 (2015).

[19] D. Vivarès, L. Belloni, A. Tardieu, and F. Bonneté, Eur. Phys. J. E 9, 15 (2002). 
[20] R. Tuinier, J. K. G. Dhont, and C. G. D. Kruif, Langmuir 16, 1497 (2000).

[21] P. G. Bolhuis, E. J. Meijer, and A. A. Louis, Phys. Rev. Lett. 90, 068304 (2003).

[22] I. Yadav, S. Kumar, V. K. Aswal, and J. Kohlbrecher, Phys. Rev. E 89, 032304 (2014).

[23] K. Rezwan, L. P. Meier, and L. J. Gauckler, Biomaterials 26, 4351 (2005).

[24] B. Bharti, J. Meissner, S. H. L. Klapp, and G. H. Findenegg, Soft Matter 10, 718 (2014).

[25] I. Yadav, V. K. Aswal, and J. Kohlbrecher, Phys. Rev. E 91, 052306 (2015).

[26] S. Mehan, V. K. Aswal, and J. Kohlbrecher, Langmuir 30, 9941 (2014).

[27] P. Roach, D. Farrar, and C. C. Perry, J. Am. Chem. Soc. 128, 3939 (2006).

[28] P. Asuri, S. S. Karajanagi, H. C. Yang, T. J. Yim, R. S. Kane, and J. S. Dordick, Langmuir 22, 5833 (2006).

[29] E. Casals, T. Pfaller, A. Duschl, G. J. Oostingh, and V. Puntes, ACS Nano 4, 3623 (2010).

[30] M. E. A. Tam and K. H. Schifferli, Biomed. Mater. 3, 034001 (2008).

[31] I. Lynch, T. Cedervall, M. Lundqvist, C. C. Lago, S. Linse, and K. A. Dawson, Adv. Colloid Interface Sci. 134-135, 167 (2007).

[32] Y. Zeng, S. Grandner, C. L. P. Oliveira, A. F. Thunemann, O. Paris, J. S. Pedersen, S. H. L. Klapp, and R. V. Klitzing, Soft Matter 7, 10899 (2011).

[33] A. Albanese, P. S. Tang, and W. C. W. Chan, Annu. Rev. Biomed. Eng. 14, 1 (2012).

[34] A. J. Chinchalikar, V. K. Aswal, J. Kohlbrecher, and A. G. Wagh, J. Nanofluids 2, 194 (2013).

[35] W. Jiang, B. Y. S. Kim, J. T. Rutka, and W. C. W. Chan, Nat. Nanotechnol. 3, 145 (2008).

[36] M. Lundqvist, J. Stigler, G. Elia, L. Iseult, T. Cedervall, and K. A. Dawson, Proc. Natl. Acad. Sci. USA 105, 14265 (2008).

[37] S. Kumar, V. K. Aswal, and P. Callow, Langmuir 30, 1588 (2014).
[38] C. D. Walkey, J. B. Olsen, H. Guo, A. Emili, and W. C. W. Chan, J. Am. Chem. Soc. 134, 2139 (2012).

[39] S. M. Ilett, A. Orrock, W. C. K. Poon, and P. N. Pusey, Phys. Rev. E 51, 1344 (1995).

[40] B. Gotzelmann, R. Evans, and S. Dietrich, Phys. Rev. E 57, 6785 (1998).

[41] K. Park, H. Koerner, and R. A. Vaia, Nano Lett. 10, 1433 (2010).

[42] R. A. Schoonheydt, Chem. Soc. Rev. 39, 5051 (2010).

[43] S. A. R. Kynde, N. S. Gislinge, M. C. Pedersen, S. R. Midtgaard, J. B. Simonsen, R. Schweins, K. Mortensen, and L. Arleth, Acta Crystallogr., Sect. D: Biol. Crystallogr. 70, 371 (2014).

[44] R. Pecora, Dynamic Light Scattering (Plenum, New York, 1985).

[45] J. B. Hayter and J. Penfold, Colloid Polym. Sci. 261, 1022 (1983).

[46] Y. Liu, W.-R. Chen, and S.-H. Chen, J. Chem. Phys. 122, 044507 (2005).

[47] J. Kohlbrecher and W. Wagner, J. Appl. Crystallogr. 33, 804 (2000).

[48] C. G. Windsor, J. Appl. Crystallogr. 21, 582 (1988).

[49] B. J. Frisken, Appl. Opt. 40, 4087 (2001).

[50] P. A. Hassan, S. Rana, and G. Verma, Langmuir 31, 3 (2015).

[51] F. Zhang, M. W. A. Skoda, R. M. J. Jacobs, R. A. Martin, C. M. Martin, and F. Schreiber, J. Phys. Chem. B 111, 251 (2007).

[52] J. S. Pedersen, D. Posselt, and K. Mortensen, J. Appl. Crystallogr. 23, 321 (1990).

[53] V. K. Aswal and P. S. Goyal, Curr. Sci. 79, 947 (2000).

[54] A. Shukla, E. Mylonas, E. Di Cola, S. Finet, P. Timmins, T. Narayanan, and D. I. Svergun, Proc. Natl. Acad. Sci. USA 105, 5075 (2008).

[55] S. Asakura and F. Oosawa, J. Polym. Sci. 33, 183 (1958).

[56] H. N. W. Lekkerkerker, W. C. Poon, P. N. Pusey, A. Stroobants, and P. B. Warren, Europhys. Lett. 20, 559 (1992).

[57] S. Kumar, M.-J. Lee, V. K. Aswal, and S.-M. Choi, Phys. Rev. E 87, 042315 (2013).

[58] J. C. Crocker and D. G. Grier, Phys. Rev. Lett. 77, 1897 (1996).

[59] A. J. Chinchalikar, V. K. Aswal, J. Kohlbrecher, and A. G. Wagh, Phys. Rev. E 87, 062708 (2013). 